\title{
Impact de la trituration et du raffinage sur la teneur en micronutriments des huiles végétales de colza, soja et tournesol
}

\author{
Jennifer Régis ${ }^{1}$, Florent Joffre ${ }^{2}$ et Frédéric Fine ${ }^{1, \star}$ \\ 1 Terres Inovia, 11 rue Monge, 33600 Pessac, France \\ 2 ITERG, 11 rue Monge, 33600 Pessac, France
}

Reçu le 22 janvier 2016 - Accepté le 11 février 2016

\begin{abstract}
Résumé - Majoritairement constituées de triglycérides et de leurs acides gras, les huiles végétales contiennent également entre 1 et $5 \%$ de micronutriments tels que les tocophérols, les phytostérols, les polyphénols et les coenzymes Q. Naturellement présents dans les graines de colza, soja et tournesol, ces composés sont connus pour leurs effets bénéfiques sur la santé. Leur transfert et leur préservation de la graine jusqu'à l'huile apparaissent dès lors comme primordiaux. Pourtant, une grande part de ces micronutriments est partiellement à totalement détruite au cours des procédés de trituration et de raffinage conventionnels. La préparation des graines (aplatissage, broyage, cuisson), l'extraction par solvant et le raffinage des huiles (neutralisation, décoloration et désodorisation) sont, en effet, autant d'étapes qui ont une influence significative sur les teneurs en constituants mineurs des huiles brutes et raffinées. Des travaux visant à démontrer l'efficacité de différentes méthodes alternatives ont dès lors été conduits.
\end{abstract}

Mots clés : Trituration / raffinage / méthodes alternatives / micronutriments

\begin{abstract}
The impact of crushing and refining processes on micronutrient content in rapeseed, soybean and sunflower oils. Mainly composed of triglycerides and their fatty acids, vegetable oils also contain between 1 and 5\% of micronutrients such as tocopherols, phytosterols, polyphenols and coenzymes Q. Naturally present in rape, soya and sunflower seeds, these compounds are known for their benefits to health. Their transfer and their preservation from seed to oil appear as essential. However, much of these micronutrients are partially to totally destroyed during crushing and refining processes. Seed preparation (rolling, grinding, cooking), solvent extraction and purification of oils (neutralization, bleaching and deodorization) are, indeed, all steps that have a significant influence on levels of minor components of crude and refined oils. Work to demonstrate the efficiency of different alternative methods have then been conducted.
\end{abstract}

Keywords: Crushing / refining / alternative methods / micronutrients

\section{Introduction}

En 2012, la production mondiale d'huiles végétales a atteint 186,4 millions de tonnes. Plus de $70 \%$ de cette production ont été assurés par seulement cinq oléagineux : palme (29\%), soja (22\%), colza (13\%), tournesol (8\%) et olive $(2 \%)$. Plus de $70 \%$ de cette production mondiale est utilisée par l'alimentation soit consommée directement (huiles de table, de cuisson) soit transformée par l'industrie agroalimentaire (margarine, plats cuisinés, sauces, biscuits, aliments frits ...) (PROLEA, 2012).

\footnotetext{
^ Correspondance : f.fine@terresinovia.fr
}

Les huiles végétales brutes sont produites au cours du procédé de trituration des graines oléagineuses. Cette opération, qui n'a que relativement peu évoluée depuis 60 ans, comporte trois étapes principales (Fig. 1) (Bauer et al., 2010; Evrard et al., 2007 ; Fine et al., 2013) :

La préparation des graines : Les graines oléagineuses sont tamisées à l'aide de tamis rotatifs afin de retirer les impuretés qui pourraient affecter la qualité des huiles et des tourteaux et complexifier les opérations unitaires à venir. Un chauffage à $60{ }^{\circ} \mathrm{C}$ puis un aplatissage de ces graines au travers de deux cylindres sont ensuite réalisés pour favoriser la libération de l'huile au cours des étapes de pression et d'extraction. Cette étape d'aplatissage est cruciale puisque l'huile contenue dans 


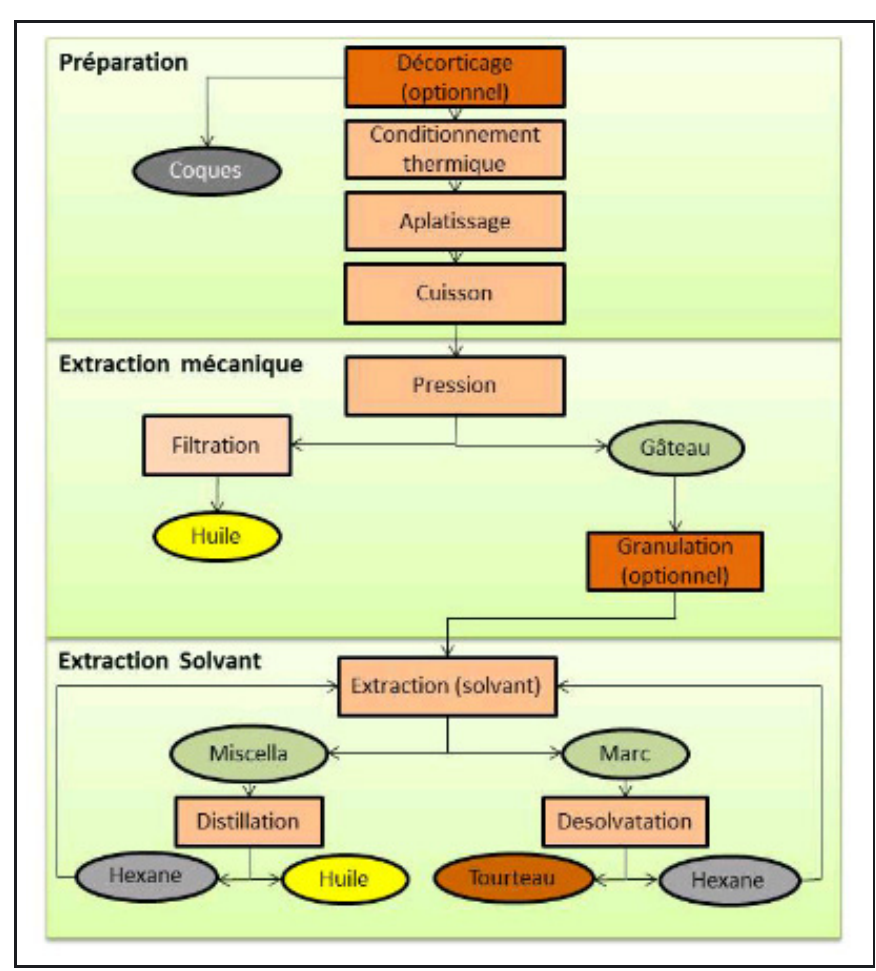

Fig. 1. Opérations unitaires du procédé de trituration (Source : Fine et al., 2013).

une graine non ou mal aplatie ne pourra pas être extraite. Les flocons obtenus sont cuits entre 80 et $100{ }^{\circ} \mathrm{C}$ afin d'inactiver certaines enzymes, de dégrader les composés entourant les zones de stockage des lipides et améliorer ainsi l'efficacité du pressage.

L'extraction mécanique : Le passage de ces flocons cuits au travers d'une presse continue à vis permet d'extraire environ $50 \%$ de l'huile initialement contenue dans les graines. Les matières solides potentiellement contenues dans cette huile sont alors retirées par tamisage, filtration ou décantation. Le gâteau ou écailles de pression, qui contient encore entre 15 et $20 \%$ d'huile, est ensuite granulé pour faciliter la percolation du solvant lors de l'étape d'extraction.

L'extraction par solvant : L'épuisement du gâteau se déroule dans un extracteur fonctionnant en continu par immersion ou par percolation et dans lequel de l'hexane circule à contre-courant. Le solvant qui s'est continuellement enrichi en huile au cours de l'extraction, est alors distillé tandis que le marc est désolvanté par évaporation de l'hexane dans un désolvanteur.

Les huiles brutes de pression et d'extraction sont ensuite mélangées puis raffinées afin de fournir aux consommateurs une huile de qualité, exempte d'impuretés et de contaminants, et qui réponde aux exigences réglementaires. Le raffinage chimique des huiles comporte quatre étapes (Fig. 2) (Devillers et al., 2010 ; Evrard et al., 2007).

Dégommage : Cette opération, qui consiste en l'ajout d'acide et/ou d'eau aux huiles brutes, permet d'éliminer les 0,2 à $1,8 \%$ de phospholipides qu'elles contiennent. Ainsi, de 0,1 à $0,3 \%$ d'acide phosphorique à $75 \%$ sont mélangés aux huiles brutes de colza et de tournesol préalablement chauffées entre 60 et $80{ }^{\circ} \mathrm{C}$. En revanche, seuls environ $3 \%$ d'eau sont ajoutés à l'huile brute de soja. Après mélangeage, centrifugation et séchage des gommes formées, la lécithine est récupérée afin d'être valorisée.

Neutralisation : Les acides gras libres, dont les teneurs dans les huiles varient entre 0,3 et $5 \%$, sont extraits au cours de la neutralisation. Cette étape du procédé consiste en l'ajout d'une solution de soude, dont la concentration dépend de l'acidité de l'huile, aux mélanges issus du dégommage. Après mélangeage et centrifugation, les pâtes de neutralisation formées sont lavées à l'eau à plusieurs reprises avant d'être séchées sous vide.

Décoloration: Dans un décolorateur contenant de l'huile à 90-110 ${ }^{\circ} \mathrm{C}$ sont introduits de 0,2 à $2 \%$ d'agents d'adsorption tels que les terres décolorantes. Après une mise en contact de 30 min sous agitation et sous vide poussé, l'huile est refroidie puis filtrée afin d'en extraire les pigments encore présents.

Désodorisation : Les composés volatils contenus dans l'huile à $180-240^{\circ} \mathrm{C}$ sont enlevés par entraînement à la vapeur d'eau sous vide très poussé.

\section{Les graines de colza, tournesol et soja : une source de micronutriments d'intérêt}

Les graines de colza, soja et tournesol sont une source d'acides gras insaturés, les oméga 9, 6 et 3, dont l'intérêt nutritionnel fait l'objet d'un très grand nombre de publications. Toutefois, ces graines contiennent également des micronutriments présents en quantité plus faible mais également connus pour leurs bienfaits sur la santé. Citons par ordre d'importance massique dans les huiles raffinées les phytostérols, qui font l'objet d'une allégation santé, les tocophérols, qui font l'objet d'une allégation nutritionnelle et enfin les coenzymes Q. Enfin, dans les graines et les huiles brutes la présence de polyphénols est également un atout santé. Les teneurs en micronutriments dans les graines dépendent fortement de l'espèce végétale considérée et des conditions pédoclimatiques. Une très grande diversité de résultats est alors relevée dans la littérature (Fine et al., 2015); l'absence d'information sur l'origine des variétés, les conditions de culture et les méthodes d'analyse expliquent en partie cette grande diversité. Le tableau 1 reprend les données analytiques obtenues dans le cadre du Projet Européen Optim'Oils. Il ressort de ces données une grande dispersion des résultats qui complique l'exploitation des données de transfert et d'élimination lors du process huilier.

Les phytostérols sont des alcools triterpéniques tétracycliques regroupant de multiples composés différents dont le chef de file est le $\beta$-sitostérol. Les graines de colza, soja et tournesol contiennent respectivement en moyenne 2761, 350 et $1465 \mathrm{mg}$ stérols totaux $/ \mathrm{kg}$ de graine (Tab. 1). L'absorption de 1,5 à $3 \mathrm{~g}$ de ces composés a été associée à une réduction de taux de cholestérol sanguin (Fine et Joffre, 2015). Cette allégation santé est reprise dans différents produits de grande consommation comme des margarines ou des boissons.

Les tocophérols, plus communément appelés la vitamine E, regroupent 8 molécules différentes, 4 tocophérols et 4 tocotriénols. La présence d'un cycle phénolique leur confère des 


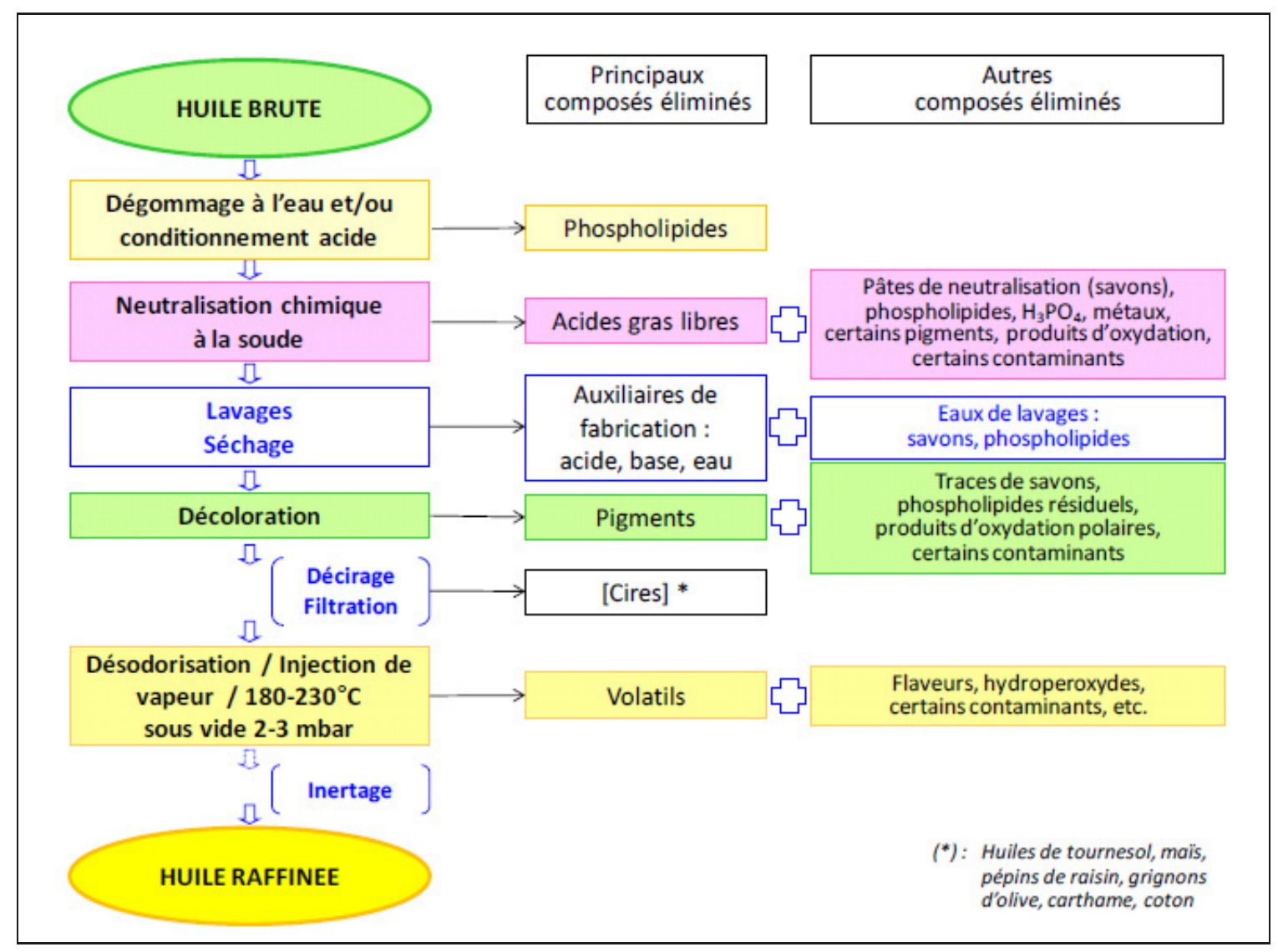

Fig. 2. Raffinage des huiles par voie chimique (Source : Devillers et al., 2010).

Tableau 1. Teneurs en phytostérols, Vitamine E, CoQ9 + 10 et Polyphénols totaux présents dans des graines de tournesol, de colza et de soja de variétés et de lieux de production différents (ITERG, Projet Optim’oils; Données non publiées). Les résultats sont exprimés sous forme de moyenne (min-max).

\begin{tabular}{|c|c|c|c|}
\hline Graines & $\begin{array}{c}\text { Tournesol } \\
\text { (33 échantillons) }\end{array}$ & $\begin{array}{c}\text { Colza } \\
\text { (18 échantillons) }\end{array}$ & $\begin{array}{c}\text { Soja } \\
\text { (9 échantillons) }\end{array}$ \\
\hline Phytostérols & $1465(980-2101)$ & $2761(2040-3436)$ & $350(275-489)$ \\
\hline Vitamine E & $233(117-305)$ & $246(172-325)$ & $106(44-190)$ \\
\hline CoQ9 + CoQ10 & $20(14-27)$ & $43(30-57)$ & $13(10-15)$ \\
\hline Polyphénols totaux & $6863(4143-9158)$ & $6141(4547-7077)$ & 4154 (1771-6627) \\
\hline
\end{tabular}

propriétés antioxydantes que ce soit dans les huiles, les aliments mais également une fois ingérées une efficacité contre le stress oxydatif. Cet impact lui permet de bénéficier d'une allégation nutritionnelle (Fine et Joffre, 2015). Des teneurs variables sont relevées dans les graines; $172-325 \mathrm{mg} / \mathrm{kg}$ de graines de colza, 44-190 mg/kg de graines de soja et $117-$ $305 \mathrm{mg} / \mathrm{kg}$ de graines de tournesol. Cette variabilité dépend principalement des conditions pédoclimatiques notamment l'ensoleillement ou le stade de maturité.

Les polyphénols regroupent plus de 8000 molécules différentes, de l'acide phénolique simple au tanin hautement polymérisé. Tous ces composés ont des rôles variés; ils sont responsables de la couleur (anthocyane), de l'odeur (phénols volatils), du goût (astringence, amertume) des végétaux, ils interviennent dans les systèmes de défense de la plante, ce sont de puissants antioxydants aux nombreux effets santé. Ils sont présents en grande quantité dans les graines de colza
(4547-7077 mg/kg de graine), soja (1771-6627 mg/kg de graine) et tournesol (4143-9158 mg/kg de graine).

Les coenzymes Q9 et Q10 sont constituées d'un cycle phénolique sur lequel est greffée une chaîne contenant 9 ou 10 répétitions d'un cycle isoprénique. Deux rôles majeurs ont été mis en évidence : la coenzyme Q10 est une molécule clé de la chaîne respiratoire mitochondriale et sous sa forme réduite circulante elle présente des propriétés anti-oxydantes importantes. Faiblement présents dans les graines de colza (30$57 \mathrm{mg} / \mathrm{kg}$ de graine), soja (10-15 mg/kg de graine) et tournesol (14-27 mg/kg de graine), ces micronutriments dont l'effet antioxydant a été rapporté dès l'absorption de 90 à 100 mg/j, sont principalement apportés par les poissons et les viandes rouges (Fine et Joffre, 2015). Enfin contrairement aux autres micronutriments étudiés dans cet article, ils ne sont pas uniquement apportés par l'alimentation mais au contraire leur synthèse endogène couvre les besoins métaboliques. Toutefois cette synthèse 
est fortement diminuée avec l'âge ou avec certains traitements médicamenteux comme les statines, ce qui renforce l'attrait des apports alimentaires en coenzyme Q10.

\section{Des graines oléagineuses aux huiles brutes : la trituration}

\subsection{Impact de la trituration sur les teneurs en micronutriments des huiles brutes}

Le transfert des tocophérols des graines aux huiles brutes dépend fortement des prétraitements et des procédés mis en œuvre. Chu et Lin (1993) ont montré que l'aplatissage et le broyage de graines de soja conduisent à la perte de ces micronutriments suite à la dégradation de ces derniers par des réactions enzymatiques. De plus, d'après Tasan et al. (2011), les huiles brutes de tournesol obtenues par le procédé conventionnel (cuisson, pression) ont des teneurs en vitamine $\mathrm{E}$ plus faibles que celles obtenues par extrusion simple (507 vs. $689 \mathrm{mg} / \mathrm{kg}$ ). Au cours du procédé de trituration des graines, les phénols restent très majoritairement piégés dans le tourteau et ne sont donc pas disponibles pour la consommation humaine. De nombreux travaux visant à étudier l'impact de la trituration sur les teneurs en phénols des huiles de colza ont par conséquent été menés (Fine et al., 2015). Dans la graine de colza, il a été montré que les prétraitements à la vapeur favorisaient la formation de canolol, composé issu de la décarboxylation de la sinapine. Or le canolol a un meilleur taux de transfert dans l'huile que son précurseur, enrichissant de fait les huiles brutes obtenues. En revanche, l'influence d'une élévation de la température est peu significative dans le cas du tournesol et n'a pas été étudiée dans le cas du soja. Enfin dans le cadre d'une étude récente, sur la comparaison de procédés conventionnels sur des graines de colza et de tournesol (aplatissage, cuisson, pression $v s$. aplatissage, cuisson, pression + extraction à l'hexane des tourteaux de pression), peu ou pas de différences ont été observées : +5 à $+6 \%$ de stérols et 0 à $+8 \%$ de vitamine $E$ dans les huiles de pression + extraction. L'utilisation de l'hexane permet non seulement d'augmenter les rendements d'extraction en huile mais également les quantités de micronutriments (Fine et Joffre, 2015).

\subsection{Procédés alternatifs}

Bien que les procédés de trituration des graines conventionnels aient une influence relativement faible sur les teneurs en micronutriments d'intérêt des huiles brutes, plusieurs procédés alternatifs visant à réduire leur perte et à améliorer leur conservation sont aujourd'hui testés (Fine et al., 2015). Ainsi, il est apparu que la substitution de l'hexane par des solvants extracteurs alternatifs tels que l'éthanol conduisait à un enrichissement des huiles brutes en stérols $(+20 \%)$, vitamine $\mathrm{E}$ $(+50 \%)$ et polyphénols $(+550 \%)$ (ITERG, Projet Optim'oils ; données non publiées).

Il peut de plus être noté qu'une amélioration des rendements en huile et une augmentation des teneurs en polyphénols, coenzymes Q10 et vitamine E ont aussi été remarquées suite à l'application de micro-ondes sur des pépins de raisin. En effet, après réalisation d'un prétraitement thermique à $110^{\circ} \mathrm{C}$, les huiles de pression renfermaient $138,9 \mathrm{mg}$ par eq ac gallic/kg d'huile de polyphénols totaux, 63,4 mg/kg de coenzymes Q10 et $552 \mathrm{mg} / \mathrm{kg}$ de vitamine E. Par rapport à un prétraitement thermique à $60{ }^{\circ} \mathrm{C}$, cela représente des augmentations respectives de $90 \%, 33 \%$ et $6 \%$.

Enfin, l'extraction enzymatique en milieu aqueux de graines de tournesol permet d'améliorer les teneurs en stérols, polyphénols et coenzyme Q9 mais sans impact sur les tocophérols (Fine et al., 2015).

\section{Le raffinage des huiles végétales brutes : impact sur les teneurs en micronutriments des huiles raffinées}

De nombreuses études ont montré qu'une part importante des micronutriments des huiles brutes était détruite au cours des étapes du raffinage (Ferrari et al., 1996). D'après la littérature (Fine et al., 2015), le raffinage entraînerait des pertes en tocophérols allant de $15-51 \%, 0-82 \%$ et $32-38 \%$ pour des huiles brutes de colza, soja et tournesol respectivement. Les étapes de neutralisation alcaline et de désodorisation sont généralement les plus destructrices vis-à-vis de ces composés. Pour les stérols, ces pertes, qui se produisent au cours de la désodorisation, sont très variables allant de 29-51\%, $20 \%$ et $19-38 \%$ pour les huiles de colza, soja et tournesol respectivement.

La neutralisation est l'étape la plus critique pour les phénols qui sont éliminés presque intégralement. Cela s'explique par leur polarité et leur hydrophilicité. Dans ce contexte, certains phénols simples comme le canolol, moins polaire que son précurseur l'acide sinapique, peuvent être en partie préservés. Malheureusement, leur faible poids moléculaire conduit à leur élimination par distillation ou par destruction thermique lors de l'étape de désodorisation.

La grande variabilité d'effets observés et décrits dans la littérature dépend principalement de la méthodologie et des modèles d'études utilisés. En effet, des travaux réalisés à l'échelle laboratoire sur moins de $100 \mathrm{~g}$ d'huile ou à l'échelle pilote sur plusieurs kilogrammes ne donnent pas des résultats identiques. De plus, même à l'échelle pilote où le raffinage est réalisé « en batch » un biais technologique est présent par comparaison avec le raffinage en continu pratiqué par les industriels. Sur la base d'un essai de raffinage d'huile de colza en condition industrielle, nous avons quantifié des pertes en micronutriments beaucoup moins importantes : $5 \%$ des tocophérols, $8 \%$ des stérols et $2 \%$ des coenzymes Q10 (données non publiées).

\section{Conclusion}

Les opérations de trituration et de raffinage ont respectivement pour objectif de produire de l'huile avec un fort rendement tout en préservant les qualités nutritionnelles des tourteaux pour pouvoir les valoriser dans le secteur de l'alimentation animale, et d'assurer la qualité et la sécurité sanitaire des huiles destinées à la consommation humaine. Connus pour avoir des effets bénéfiques sur la santé, les stérols, les tocophérols, les phénols et les coenzymes Q9 et Q10 sont partiellement à totalement détruits au cours de ces procédés 
Tableau 2. Impact de la trituration et du raffinage sur les teneurs en micronutriments des huiles brutes et raffinées (Fine et al., 2015).

\begin{tabular}{|c|c|c|c|c|c|c|c|}
\hline & \multicolumn{3}{|c|}{$\begin{array}{l}\text { Pourcentage de micronutriments } \\
\text { transférés au cours de la trituration }\end{array}$} & \multicolumn{4}{|c|}{$\begin{array}{l}\text { Pourcentage de micronutriments éliminés } \\
\text { au cours du raffinage }\end{array}$} \\
\hline & Colza & Soja & Tournesol & Colza & Soja & Tournesol & Étape la plus destructrice \\
\hline Stérols & \multicolumn{3}{|c|}{ Données analytiques non concluantes } & $29-51$ & $\approx 20$ & $19-38$ & Désodorisation \\
\hline Tocophérols & \multicolumn{3}{|c|}{ Données analytiques non concluantes } & $15-51$ & $0-82$ & $32-38$ & Désodorisation \\
\hline Coenzymes Q & \multicolumn{3}{|c|}{ Données analytiques non concluantes } & \multicolumn{3}{|c|}{ Données analytiques non concluantes } & Désodorisation et Neutralisation \\
\hline Polyphénols & 4-5 & $\approx 1$ & $2-7$ & \multicolumn{3}{|c|}{ Perte totale } & Neutralisation \\
\hline
\end{tabular}

(Tab. 2, Fine et al., 2015). Le recours à des méthodes alternatives visant à diminuer leur perte et/ou améliorer leur extraction a alors été envisagé. Les résultats obtenus semblent prometteurs dans la mesure où une meilleure préservation de ces micronutriments a été constatée.

Remerciements. Ce travail a été réalisé, en partenariat avec la SAS PIVERT, dans le cadre de l'Institut pour la Transition Energétique (ITE) P.I.V.E.R.T. (www.institut-pivert.com) retenu parmi les Investissements d'Avenir. Ce travail a bénéficié d'une aide de l'Etat au titre du Programme d'Investissements d'Avenir portant la référence ANR-001-01.

\section{Références}

Bauer WJ, Badoud R, Löliger J. 2010. «Les lipides », In Principes de chimie des constituants et de technologie des procédés. Presses polytechniques et universitaires romandes, Lausanne, Alain Etournaud.

Chu YH, Lin JY. 1993. Factors affecting the content of tocopherol in soybean oil. J. Am. Oil Chem. Soc. 70: 1263-1268.

Devillers PH, Thébault J, Mathellier B, et al. 2010. Huiles végétales : Guide d'aide à l'application des meilleures technologies disponibles (document électronique), http://iterg.com/IMG/pdf/guide_ mtd_iterg_2.pdf.
Evrard J, Pagès-Xatart-Pares X, Argenson C, Morin O. 2007. Procédés d'obtention et compositions nutritonnelles des huiles de tournesol, olive et colza. Cah. Nutr. Diét. 42: 1S13-1S23.

Ferrari RA, Schulte E, Esteves W, Brühl L, Mukherjee KD. 1996. Minor constituents of vegetable oils during industrial procesing. J. Am. Oil Chem. Soc. 73: 587-592.

Fine F, Joffre F. 2015. Impact de la trituration et du raffinage sur la teneur en micronutriments des huiles végétales, Communication présentée au cours de la journée GLN sur le thème : consommations et fonctionalités des lipides. Nouveaux horizons, Paris, France.

Fine F, Abert Vian M, Fabiano Tixier AS, et al. 2013. Les agrosolvants pour l'extraction des huiles végétales issues de graines oléagineuses. OCL 20: A502.

Fine F, Brochet C, Gaud M, et al. 2015. Micronutrients in vegetable oils : The impact of crushing and refining processes on vitamins and antioxydants in sunflower, rapeseed and soybean oils. Eur. J. Lipid Sci. Technol. 117: 1-18.

PROLEA. Statistiques des oléagineux et protéagineux, Paris, 2012 (document électronique), http://www. prolea.com/fileadmin/internet/fichiers/chiffres/ de-la-production-a-la-consommation-2012-2013.pdf.

Tasan M, Gecgel U, Demirci M. 2011. Effects of storage and industrial oilseed extraction methods on quality and stability characteristics of crude sunflower oil (Helianthus annuus L). Grasas y aceites 62: 389-398.

Cite this article as: Jennifer Régis, Florent Joffre, Frédéric Fine. Impact de la trituration et du raffinage sur la teneur en micronutriments des huiles végétales de colza, soja et tournesol. OCL 2016, 23(3) D302. 